\title{
Taninos condensados de la esparceta y su efecto sobre los parámetros de la fermentación ruminal. Influencia del estado fenológico y de la conservación. Revisión bibliográfica
}

\author{
Pablo José Rufino-Moya ${ }^{1,2}$, Mireia Blanco ${ }^{1,2}$ y Margalida Joy ${ }^{1,2, *}$ \\ 1 Unidad de Producción y Sanidad Animal. Centro de Investigación y Tecnología Agroalimentaria de \\ Aragón (CITA). Avda. Montañana 930, 50059, Zaragoza, España \\ 2 Instituto Agroalimentario de Aragón - IA2 (CITA-Universidad de Zaragoza). Calle Miguel Servet 177, \\ 50013, Zaragoza, España
}

\begin{abstract}
Resumen
La esparceta (Onobrychis viciifolia) es una leguminosa forrajera con taninos condensados (TC), cuyo contenido puede variar en función de la variedad, el estado fenológico, la conservación del forraje, etc. Los TC pueden afectar a algunos parámetros de la fermentación ruminal dependiendo de su contenido en la dieta y la composición química de ésta, de la estructura química de los TC y de la especie de rumiante, entre otros. En esta revisión se pretende estudiar la influencia de la presencia de los TC, del estado fenológico y de la conservación de la esparceta en el contenido y fracciones de TC (libres, ligados a proteína y a fibra), así como en sus características estructurales (grado de polimerización, relación prodelfinidina:procianidina y relación cis:trans en la unidad de flavanol) y sus efectos en la fermentación ruminal de ovino y vacuno. Los TC provocan principalmente una reducción de la producción de gas y metano y una reducción de la degradación de la proteína que se manifiesta por una reducción en los contenidos de amoniaco e iso-ácidos. Sin embargo, existen discrepancias en los resultados obtenidos tanto en los estudios que usan polietilenglicol, como en los estudios que evalúan el efecto del estado fenológico y la conservación. Es difícil diferenciar si los cambios que se dan son debidos a cambios en la composición química o cambios en los TC y sus fracciones. A lo largo del desarrollo del forraje se puede dar una reducción del contenido de TC mientras que la conservación da lugar a cambios en la distribución de las fracciones de TC, especialmente en el ensilado. Sin embargo, los estudios no son concluyentes y muestran que otros factores distintos al contenido en TC, como el tipo de dieta, la especie rumiante o el tiempo de incubación, también afectan a la fermentación ruminal.
\end{abstract}

Palabras clave: Fermentación in vitro, metano, amoniaco, ácidos grasos volátiles, heno, silo.

Condensed tannins of sainfoin and their effect on ruminal fermentation. Influence of phenological stage and preservation. A review

\footnotetext{
Abstract

Sainfoin (Onobrychis viciifolia) is a forage legume with condensed tannins (CT), varying the content upon different factors such as the variety, phenological stage, forage preservation, etc. The CT can affect some

* Autor para correspondencia: mjoy@cita-aragon.es

Cita del artículo: Rufino-Moya PJ, Blanco M, Joy M (2021). Taninos condensados de la esparceta y su efecto sobre los parámetros de la fermentación ruminal. Influencia del estado fenológico y de la conservación. Revisión bibliográfica. ITEA-Información Técnica Económica Agraria 117(3): 227-246. https://doi.org/10.12706/itea.2020.026
} 
parameters of the ruminal fermentation depending on their content and their chemical structure, the chemical composition of the diet and the ruminant species, among other factors. The objective of this review is to study the influence of $C T$, the phenological stage and the preservation of the sainfoin on the CT content and its fractions (free, protein-bound and fibre-bound), as well as in its structural characteristics (degree of polymerization, ratio of prodelphinidin:procyanidin and ratio of cis:trans in the flavanol unit) and their effects on ruminal fermentation in sheep and cattle. Condensed tannins mainly cause a reduction in the production of gas and methane and a reduction in the degradation of the protein that is showed by a reduction in the contents of ammonia and iso-acids. However, there are still controversies in the studies using polyethylene glycol and in the studies that evaluate the phenological stage and preservation. It is difficult to ascribe the difference to changes in chemical composition and/or changes in CTs characteristics... As forage develops, a reduction in CT content can occur whereas preservation leads to changes in the distribution of CT fractions, especially in silage. However, the studies are not conclusive and show that other factors different to the CT content also affect the ruminal fermentation, such as type of diet, ruminant species or incubation time.

Keywords: In vitro fermentation, methane, ammonia, volatile fatty acids, hay, silage.

\section{Introducción}

Algunas leguminosas forrajeras tienen taninos condensados (TC), que son compuestos secundarios cuya función principal es la defensa de la planta (Bodas et al., 2012). Entre las especies de leguminosas forrajeras con TC cultivadas en España destaca la esparceta (Onobrychis viciifolia) con una superficie cultivada de más de 25.000 Ha y una producción de unas 260.000 toneladas en 2018 (MAPA, 2019). La producción anual de la esparceta se concentra principalmente en primavera, produciéndose alrededor de dos tercios del total, y el resto se distribuye entre el verano y el otoño (Delgado et al., 2008). Esto hace que el aprovechamiento mayoritario para la alimentación de rumiantes sea mediante la conservación, siendo la henificación la forma de conservación más comúnmente usada. Dicha henificación conlleva una modificación de la composición química con pérdidas de proteína bruta (PB) e incremento de la fibra que pueden variar según el manejo mecánico, la exposición a la intemperie o la respiración durante el almacenamiento (Aufrere et al., 2008; Wang et al., 2015). La conservación mediante ensilado es menos frecuente por el bajo contenido en carbohidratos solubles y alta capacidad tampón de la esparceta que pueden impedir una correcta acidificación del ensilado (Heinritz et al., 2012). Sin embargo, esto se puede contrarrestar con una desecación previa al ensilado y/o la aplicación de aditivos durante el proceso de ensilado que favorecen la fermentación adecuada (Lorenz et al., 2010).

Químicamente, los TC o proantocianidinas son compuestos fenólicos oligoméricos o poliméricos no ramificados constituidos por monómeros de antocianinas (flavan-3-oles) que no son susceptibles a la degradación anaeróbica de las enzimas (Waghorn, 2008; Jonker y $\mathrm{Yu}, 2017)$. Todas las proantocianidinas contienen monómeros de (+) catequina y su isómero (-) epicatequina. Los TC se clasifican en procianidinas (PC) (constituidas únicamente por catequina y epicatequina), propelargonidinas (además contienen $(+)$ afzelequina y $(-)$ epiafzelequina) y prodelfinidinas (PD) (contienen (+) galocatequina y (-) epigalocatequina), siendo estos últimos los principales polímeros en la esparceta (Jonker y Yu, 2017; Mueller-Harvey et al., 2019). Los TC se pueden caracterizar según sus fracciones: TC libres (TCL), ligados a proteína (TCP) y ligados a fibra (TCF). Los métodos más extendidos que permiten la determinación de los TCL son el butano- $\mathrm{HCl}$ (Porter et al., 1985) y el vanillina- 
$\mathrm{HCl}$ (Broadhurst y Jones, 1978) y estos métodos modificados permiten determinar los TCP y TCF (Terrill et al., 1992). Por otro lado, se puede determinar el grado de polimerización (GP), la relación PD:PC y la relación cis:trans en la unidad del flavanol (Theodoridou et al., 2010 y 2011a) mediante técnicas cromatográficas como la tiólisis o floroglucinólisis (Gea et al., 2011) o con equipos de resonancia magnética nuclear, MALDI TOF MS o UPLCMS/MS (Stringano et al., 2011; Engstrom et al., 2014). Dichas características determinan las propiedades químicas y la bioactividad de los TC, influyendo en la formación de complejos con la proteína y la fibra por parte de los TC (Jonker y Yu, 2017). Sin embargo, la cuantificación y caracterización es complicada debido a una falta de normalización de los métodos analíticos para la determinación de TC, así como de los estándares utilizados para expresarlos, generando una gran variabilidad de resultados entre estudios (Frutos et al., 2004a; Toral et al., 2016).

El contenido de TC y sus características estructurales dependen tanto de factores intrínsecos de la planta (variedad, estado fenológico,...) (Theodoridou et al., 2011b; Hatew et al., 2015) como de factores extrínsecos (humedad, temperatura, radiaciones solares, conservación,...) (Mole et al., 1988; McMahon et al., 2000; Li et al., 2014). Diversos estudios apuntan a que los TC afectan a parámetros de la fermentación ruminal, como a la producción de metano y/o la degradación ruminal de la proteína (Theodoridou et al., 2011b; Niderkorn et al., 2012). Sin embargo, los efectos sobre la fermentación ruminal dependen tanto del contenido del TC como de sus características estructurales (Theodoridou et al., 2011b; Hatew et al., 2016; Huyen et al., 2016), los cuales están influenciados por el estado fenológico y la conservación de la esparceta (Theodoridou et al., 2010; Rufino-Moya et al., 2019a,b). En la presente revisión se estudiarán los efectos del contenido en TC, del estado fenológico y de la conservación de la esparceta en el contenido y fracciones de TC (libres, ligados a proteína y a fibra), así como en sus características estructurales (grado de polimerización, relación prodelfinidina:procianidina y relación cis:trans en la unidad de flavanol) y sus efectos en la fermentación ruminal de ovino y vacuno.

\section{Presencia de taninos condensados en la esparceta y efectos sobre la fermentación ruminal}

Los factores ligados a la planta, como la variedad, influyen en el contenido y características de los TC de la esparceta (McMahon et al., 2000; Hatew et al., 2015; Jonker y Yu, 2017). El estado fenológico de la planta también determina dicho contenido (Theodoridou et al., 2011a; Rufino-Moya et al., 2019b) dado que el grado de madurez influye sobre los porcentajes de hojas, flores y frutos de una planta, los cuales presentan distinto contenido en TC (Frutos et al., 2002). Específicamente, los TC se presentan en mayor concentración en las hojas en la esparceta (Piluzza y Bullitta, 2010). Los resultados recopilados muestran una amplia variabilidad de contenido de los TC y sus fracciones, así de sus características estructurales (Tabla 1).

También hay factores medioambientales que afectan al contenido de TC (Frutos et al., 2002; Wang et al., 2015). El déficit hídrico y elevadas temperaturas (McMahon et al., 2000; Li et al., 2014), la mayor intensidad de la luz y de radiaciones solares (Mole et al., 1988) y/o las adversas propiedades físicas del suelo (Kelman y Tanner, 1990), provocan una mayor síntesis y concentración de los TC. Otro factor extrínseco de la planta que afecta al contenido de TC, es el proceso de conservación, como se detalla más adelante. 
Tabla 1. Contenido en taninos condensados totales (TCT), libres (TCL), ligados a la proteína (TCP), ligados a fibra (TCF), grado de polimerización (GP), relación de prodelfinidinas (PD) y relación de configuración cis (CIS) de la esparceta según el estado fenológico.

Table 1. Content of total condensed tannins (TCT), extractable (ECT), protein-bound (PBCT), fiber-bound $(F B C T)$, mean degree of polymerization $(m D P)$, ratio of prodelphinidins $(P D)$, ratio of cis configuration (CIS) of sainfoin according to the phenological stage.

\begin{tabular}{|c|c|c|c|c|c|c|c|c|c|}
\hline $\begin{array}{l}\text { Tipo forraje/ } \\
\text { variedad }\end{array}$ & Estado fenológico & TCT & $\mathrm{TCL}$ & TCP & TCF & GP & PD & $\mathrm{CIS}$ & Ref \\
\hline \multicolumn{10}{|l|}{ Fresco } \\
\hline \multirow[t]{3}{*}{ Ambra } & Vegetativo & 3,8 & & & & 25 & 63 & 71 & [1] \\
\hline & Botón floral & 6 & & & & 40 & 69 & 79 & [1] \\
\hline & Inicio floración & 6,2 & & & & 46 & 71 & 79 & [1] \\
\hline \multirow[t]{3}{*}{ Anatolian } & Vegetativo & 105 & & & & & & & [2] \\
\hline & Floración & 69,6 & & & & & & & [2] \\
\hline & Semilla & 42,6 & & & & & & & [2] \\
\hline \multirow[t]{3}{*}{ Esparcette } & Vegetativo & 5,2 & & & & 25 & 63,5 & 78 & [1] \\
\hline & Botón floral & 6,7 & & & & 31 & 67 & 83 & [1] \\
\hline & Inicio floración & 6,2 & & & & 39 & 70,5 & 83 & [1] \\
\hline \multirow[t]{2}{*}{ LRC 3519} & Vegetativo & & 24,5 & & & & & & [3] \\
\hline & Inicio floración & & 6,6 & & & & & & [3] \\
\hline \multirow[t]{7}{*}{ Perly } & Floración & 174 & 79 & 15 & 6 & & & & [4] \\
\hline & Final floración & 207 & 79 & 15 & 6 & & & & [4] \\
\hline & Inicio floración & 13,6 & & & & 11 & 70,5 & 81,7 & [5] \\
\hline & Final floración & 6,2 & & & & 31 & 69,2 & 82,9 & [5] \\
\hline & Inicio floración & 13,6 & & & & 11 & 70,5 & 81,7 & [6] \\
\hline & Final floración & 6,2 & & & & 31 & 69,2 & 82,9 & [6] \\
\hline & Semilla & 8,3 & & & & 21,9 & 66,2 & 79 & [6] \\
\hline \multirow[t]{3}{*}{ Reznos } & Vegetativo & 46 & 40 & 4,3 & 2,2 & & & & [7] \\
\hline & Inicio floración & 44 & 37 & 4,9 & 1,9 & & & & [7] \\
\hline & Final floración & 30 & 25 & 3,8 & 1,3 & & & & [7] \\
\hline \multirow[t]{3}{*}{ Villahoz } & Vegetativo & 3 & & & & 25 & 64 & 68 & [1] \\
\hline & Botón floral & 5 & & & & 37 & 68 & 79 & [1] \\
\hline & Inicio floración & 4,8 & & & & 37 & 70,5 & 79 & [1] \\
\hline \multirow[t]{2}{*}{ Zeus } & Vegetativo & & 35 & & & & & & [8] \\
\hline & Inicio floración & & 25 & & & & & & [8] \\
\hline \multirow[t]{2}{*}{ Nova } & Vegetativo & 67,2 & 60,8 & 5,9 & 0,5 & & & & [9] \\
\hline & Floración & 33,9 & 30 & 3,4 & 0,54 & & & & [9] \\
\hline
\end{tabular}




\begin{tabular}{|c|c|c|c|c|c|c|c|c|c|}
\hline $\begin{array}{l}\text { Tipo forraje/ } \\
\text { variedad }\end{array}$ & Estado fenológico & TCT & $\mathrm{TCL}$ & $\mathrm{TCP}$ & TCF & GP & PD & CIS & Ref \\
\hline \multicolumn{10}{|l|}{ Predesecado } \\
\hline \multirow[t]{2}{*}{ Perly } & Final floración & 166 & 69 & 22 & 9 & & & & [4] \\
\hline & Floración & 156 & 56 & 33 & 11 & & & & [4] \\
\hline \multicolumn{10}{|l|}{ Heno } \\
\hline \multirow[t]{4}{*}{ Avellinese } & Botón floral & 31,5 & & & & & & & [10] \\
\hline & Inicio floración & 9,8 & & & & & & & [10] \\
\hline & Final floración & 5,8 & & & & & & & [10] \\
\hline & Inicio semillado & 10,6 & & & & & & & [10] \\
\hline Perly & Floración & & 12,5 & & & 26,4 & 70,5 & 75,5 & [11] \\
\hline Ambra & Floración & & 8,7 & & & 30,9 & 79,3 & 74,4 & [11] \\
\hline Teruel & Floración & & 9,1 & & & 27,2 & 72,3 & 75,8 & [11] \\
\hline CPI63763 & Floración & & 8,8 & & & 20,4 & 80,6 & 70,1 & [11] \\
\hline CPI63767 & Floración & & 9 & & & 84 & 82,8 & 70,4 & [11] \\
\hline Rees A & Floración & & 15,6 & & & 19,4 & 65,7 & 76,5 & [11] \\
\hline Cotswold Common & Floración & & 10,7 & & & 15,8 & 80,8 & 75,2 & [11] \\
\hline Visnovsky & Floración & & 12 & & & 25,8 & 67,8 & 79,6 & [11] \\
\hline Cotswold Common & Floración & & 10 & & & 31 & 71 & 78 & [12] \\
\hline CPI63763 & Floración & & 7 & & & 24 & 77 & 68 & [12] \\
\hline CPI63767 & Floración & & 5 & & & 73 & 75 & 74 & [12] \\
\hline Rees A & Floración & & 11 & & & 13 & 71 & 78 & [12] \\
\hline \multicolumn{10}{|l|}{ Silo } \\
\hline \multirow[t]{3}{*}{ Perly } & Inicio floración & 33,6 & & & & & & & [13] \\
\hline & Final floración & 25,6 & & & & & & & [13] \\
\hline & Floración & 136 & 37 & 78,7 & 20,3 & & & & [14] \\
\hline
\end{tabular}

Ref $=$ Referencias.

[1] Theodoridou et al., 2011a'; [2] Bal et al., 2006²; [3]Chung et al., 201433; [4] Girard et al., 20184; [5] Theodoridou et al., 2011 b'; [6] Theodoridou et al., 2010'; [7] Rufino-moya et al., 2019b4; [8] Aufrere et al., 20082; [9] Li et al., 20144; [10] Guglielmelli et al., 20115; [11] Hatew et al., 20156; [12] Hatew et al., 20166; [13] Theodoridou et al., 2012'; [14] Brinkhaus et al., $2017^{4}$.

${ }^{1} \mathrm{~g} \mathrm{TC} / \mathrm{kg} \mathrm{MS} ;{ }^{2} \mathrm{~g}$ eq leucocianidina/kg MS; ${ }^{3} \mathrm{~g}$ eq TC Lotus pedunculatus $/ \mathrm{kg} \mathrm{MS} ;{ }^{4} \mathrm{~g}$ eq TC esparceta/kg MS; ${ }^{5} \mathrm{~g}$ eq catequina/kg MS; ${ }^{6} \mathrm{~g}$ TC esparceta/kg esparceta liofilizada. 
El efecto de los TC sobre la fermentación ruminal depende de su concentración y sus características estructurales (Hatew et al., 2016; Huyen et al., 2016), la especie rumiante que lo ingiere (Frutos et al., 2004b; Bueno et al., 2015) y la composición de la dieta y/o el tiempo de incubación (Hervás et al., 2003; Martínez et al., 2006). Las dosis elevadas de TC en la dieta (>50 g/kg MS) se asocian con efectos negativos como la reducción de la ingestión voluntaria, la digestibilidad y el metabolismo del rumen (Frutos et al., 2004a; Waghorn, 2008; Patra y Saxena, 2011). La reducción de la ingestión voluntaria debido a una alta concentración de TC está asociada con: i) la reducción de la palatabilidad debido a la sensación de astringencia (Makkar, 2003; Waghorn, 2008); ii) el enlentecimiento de la fermentación ruminal como consecuencia de la formación de complejos con los nutrientes (Kumar y Singh, 1984); iii) el desarrollo de aversiones provocadas por los efectos post-prandiales (Hervás et al., 2003). La reducción de la digestibilidad se debe: i) a la formación de complejos entre TC y los componentes de la dieta (Mueller-Harvey, 2006); ii) a la inhibición enzimática (O'Donovan y Brooker, 2001); iii) y a la acción sobre los microorganismos ruminales (Patra y Saxena, 2011). Sin embargo, los TC no tienen el mismo efecto en todos los componentes de la dieta, afectando en mayor grado a las proteínas que a los carbohidratos debido a su mayor afinidad por las primeras (Kumar y Singh, 1984; McMahon et al., 2000). Los TC provocan cambios en la fermentación ruminal, fundamentalmente en los productos finales como son el metano $\left(\mathrm{CH}_{4}\right)$, el amoniaco $\left(\mathrm{NH}_{3}-\mathrm{N}\right)$ y los ácidos grasos volátiles (AGV) (Calabrò et al., 2012; Rufino-Moya et al., 2019b).

La presencia de TC en cantidades moderadas ( $<50 \mathrm{~g} / \mathrm{kg}$ MS) puede reducir la degradación ruminal de los carbohidratos (Jayanegara et al., 2015) y de las proteínas (Aufrere et al., 2008; Mueller-Harvey, 2006). En la Tabla 2 se presenta el efecto de los TC sobre la fermentación ruminal en estudios en los cuales se ha utilizado el polietilenglicol (PEG) como agente bloqueante. EL PEG es un detergente no iónico que se usa para evaluar los efectos de los TC en el metabolismo del rumen debido a su capacidad para formar complejos con los TC y para hacer que la actividad de estos compuestos sea inocua (Makkar, 2003). Es necesario señalar que la afinidad y el efecto del PEG sobre los TC no es universal y no siempre revierte los efectos de los TC (Frutos et al., 2004b). Dicho efecto depende de diversos factores como la cantidad y el peso molecular del PEG, del contenido y la fuente de $T C$, e incluso de la especie rumiante que los consuma (Frutos et al., 2004b). La suplementación de PEG debe ser equivalente a los TC presentes en la dieta para neutralizar sus efectos (Tiemann et al., 2008), aunque se usan cantidades superiores a la relación 1:1 entre PEG:TC debido a que se considera un compuesto inerte para la función ruminal (Tiemann et al., 2008). Sin embargo, se debería ajustar la cantidad de PEG adicionado, ya que Belenguer et al. (2011) observaron que la adición de cantidades altas de PEG6000 (1 g) en incubaciones in vitro puede afectar a la comunidad microbiana ruminal. En general, la presencia de TC en la esparceta fresca no afecta a la degradabilidad in vitro de la materia seca (DIVMS) ni de la materia orgánica (DIVMO) evaluada en tiempos cortos, medios y largos (Theodoridou et al., 2011 b; Niderkorn et al., 2012; Rufino-Moya et al., 2019b), con la excepción de una disminución en dichos parámetros tras $24 \mathrm{~h}$ de incubación (Niderkorn et al., 2012). En estudios in sacco, la inclusión de PEG incrementó la degradabilidad de la materia seca a $48 \mathrm{~h}$ con esparceta fresca y ensilada e incrementó la digestibilidad de $\mathrm{MO}$ (DMO) in vivo en la esparceta dependiendo del estado fenológico (Theodoridou et al., 2010 y 2012). Scharenberg et al. (2007b) también observaron una mayor DMO en presencia de PEG cuando usaron el forraje deshidratado o en silo en estudios in vivo. 


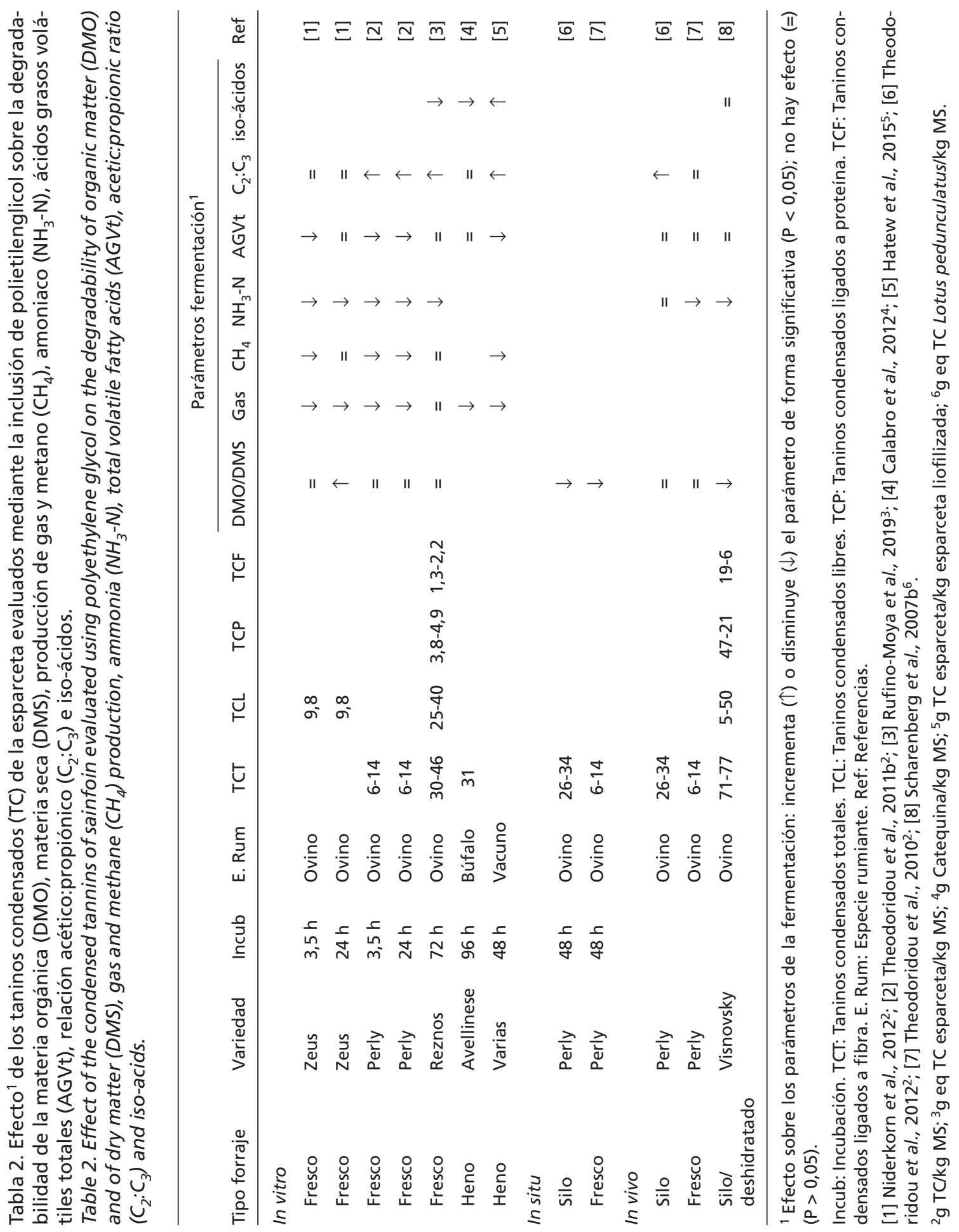


La presencia de TC provoca una reducción en la producción de gas debido a un cambio en la disponibilidad de los nutrientes, destinando una mayor proporción a la síntesis de proteína microbiana en detrimento de la empleada para la producción de AGV de cadena corta (Getachew et al., 2000). Dicho efecto se ha puesto de manifiesto en casi todos los estudios con esparceta realizados tanto en fresco como con heno a distintos tiempos de incubación (Theodoridou et al., 2011b; Calabrò et al., 2012; Niderkorn et al., 2012) (Tabla 2). Sin embargo, Rufino-Moya et al. (2019b) no encontraron dicho efecto cuando incubaron esparceta durante $72 \mathrm{~h}$, lo que pudo deberse al mayor contenido en TC de la esparceta.

Los TC pueden reducir la producción de $\mathrm{CH}_{4}$ mediante: (i) un efecto directo sobre arqueas metanogénicas ruminales, así como por una disminución del número de protozoos simbióticos asociados a la producción de metano; (ii) y un efecto indirecto sobre la producción de hidrógeno, debido a una menor degradación del alimento, fundamentalmente de fibra (Patra y Saxena, 2011; Piluzza et al., 2014). Sin embargo, el efecto de los TC sobre la producción de $\mathrm{CH}_{4}$ no es uniforme, y varía según los estudios (Tabla 2). Se ha observado una reducción de la producción de $\mathrm{CH}_{4}$ cuando se incuba in vitro esparceta fresca durante 3,5 h (Theodoridou et al., 2011b; Niderkorn et al., 2012), mientras que a $24 \mathrm{~h}$ únicamente Theodoridou et al. (2011b) observaron dicha reducción. Según Niderkorn et al. (2012), la diferente respuesta de la producción de $\mathrm{CH}_{4}$ respecto el tiempo de incubación, podría estar relacionada con que la reducción en la producción de gas a las $24 \mathrm{~h}$ estuvo asociada a una disminución de $\mathrm{CO}_{2}$ en lugar del $\mathrm{CH}_{4}$. Aunque estos mismos autores también sugieren que podría ser debido a una adaptación del ecosistema ruminal microbiano a la presencia de TC, lo cual estaría apoyado por Rufino-Moya et al. (2019b), quienes no observaron este efecto reductor de los TC de la esparceta en incubaciones de
72 h. Sin embargo, para la adaptación de los microorganimos ruminales es necesario tiempos de exposición a los TC de la esparceta más prolongados (Guglielmelli et al., 2009). Por tanto, esta discrepancia en los estudios estaría más relacionada con diferencias en el peso molecular y la estructura química de los TC, los cuales determinan los efectos sobre la producción del $\mathrm{CH}_{4}$ (Hatew et al., 2016; Huyen et al., 2016).

La presencia de TC en la dieta se ha asociado con una reducción del contenido de $\mathrm{NH}_{3}-\mathrm{N}$ debido a que reducen la solubilidad y la degradación de las proteínas de la dieta en el rumen. Además, la presencia de TC provoca un menor ritmo de fermentación que permite una mejor sincronización entre el nitrógeno y la energía, lo cual reduce el contenido en $\mathrm{NH}_{3}-\mathrm{N}$ y posibilita una mayor síntesis de proteína microbiana (Mueller-Harvey, 2006). Dicho efecto es claramente visible en los ensayos in vitro realizados con esparceta fresca a diferentes tiempos de incubación (Theodoridou et al., 2011b; Niderkorn et al., 2012; Rufino-Moya et al., 2019b). Este efecto protector de los TC de esparceta también se observa en ensayos in vivo usando esparceta fresca (Theodoridou et al., 2010) o conservada (Scharenberg et al., 2007b).

Los TC pueden reducir la producción total de los AGV y modificar sus proporciones (Hatew et al., 2015; Huyen et al., 2016), lo que está relacionado con la disminución de la DMO y/o con un aumento de la síntesis de proteína microbiana (Getachew et al., 1998; Getachew et al., 2000). El tiempo de incubación puede ser un factor determinante en la reducción de la producción total de AGV en la esparceta (Tabla 2). En este sentido usando esparceta fresca, el efecto reductor de los TC de la esparceta se manifiesta en las incubaciones in vitro cortas 3,5 h y $24 \mathrm{~h}$ (Niderkorn et al., 2012; Theodoridou et al., 2011b) pero no en incubaciones más prolongadas (Calabrò et al., 2012; Rufino-Moya et al., 2019b). 
En cuanto a la modificación de las proporciones de acético:propiónico $\left(C_{2}: C_{3}\right)$, los TC incrementan la relación en la esparceta fresca incubada in vitro durante $3,5 \mathrm{~h}$ y $24 \mathrm{~h}$ (Theodoridou et al., 2011b) o 72 h (Rufino-Moya et al., 2019b) o en un ensayo in vivo al usar la esparceta ensilada (Theodoridou et al., 2012). Sin embargo, otros estudios in vitro con tiempos similares de incubación no observaron efecto de los TC en esparceta fresca (Niderkorn et al., 2012) o henificada (Calabrò et al., 2012). Además, los TC pueden reducir los ácidos valérico e iso-ácidos (iso-butírico e iso-valérico) porque en el rumen se da la inhibición de la degradación de las proteínas (Mueller-Harvey, 2006) y/o un aumento de la utilización de los iso-ácidos para la síntesis de proteína microbiana (Martínez et al., 2006). Un efecto relevante de los TC es la reducción en la proporción del ácido valérico procedente de la valina, que tiene gran afinidad por los TC en el rumen, y de la proporción de iso-valérico. Dicho efecto fue observado por Calabrò et al. (2012), Hatew et al. (2015) y Rufino-Moya et al. (2019b). Sin embargo, en un ensayo in vivo, Scharenberg et al. (2007b) no encontraron efecto de la presencia de TC de la esparceta sobre el valérico y los iso-ácidos.

\section{Efecto del estado fenológico de la esparceta sobre el contenido en taninos condensados y la fermentación ruminal}

Los taninos condensados totales (TCT) y sus fracciones se modifican a lo largo del desarrollo del cultivo (Bal et al., 2006; Rufino-Moya et al., 2019b), aunque los datos no son concluyentes. En la Tabla 1 se presentan los contenidos en TC y sus fracciones en función del estado fenológico de la esparceta. Se ha encontrado un menor contenido al inicio (Chung et al., 2013) y al final de floración (Rufino-Moya et al., 2019b) frente al estado vegetativo. Por el contrario, Theodoridou et al. (2011a) y Girard et al. (2018) observaron un incremento de TCT durante la floración respecto al estado vegetativo, sin que existieran diferencias entre el inicio y final de floración. El contenido de TCT en la formación de la semilla se incrementa ligeramente (Theodoridou et al., 2010; Guglielmelli et al., 2011) o se reduce (Li et al., 2014) con respecto al contenido en el estado vegetativo y en la fase de floración. Esta discrepancia podría estar relacionada con las diferencias en las condiciones de cultivo, la variedad del forraje y en el estado de la semilla en el momento de la toma de muestras entre estudios.

En cuanto a las fracciones, Li et al. (2014) encontraron una reducción de los TCL y los TCP a medida que avanzaba el cultivo (estado vegetativo, de floración y con semilla madura) sin modificar los TCF. Por otro lado, RufinoMoya et al. (2019b) encontraron una reducción de los TCL y TCF al final de la floración respecto al estado vegetativo, mientras que no se encontraron diferencias en los TCP. En cambio, Girard et al. (2018) no encontraron ningún cambio en las distintas fracciones de los TC cuando compararon esparceta en estado vegetativo, en plena floración y al final de la misma.

La fermentación ruminal se puede ver afectada por el estado fenológico de la esparceta, pero es difícil discernir si se debe a la modificación de la composición química, al contenido y características de TC, o a ambos (Tabla 3). A medida que avanza el cultivo de la esparceta aumenta el contenido en fibra mientras que disminuye el contenido en TCT y la DIVMO (Aufrere et al., 2008; Theodoridou et al., 2010 y 2012; Chung et al., 2013), lo cual nos indica que el contenido en fibra es más determinante que la presencia de TC. Sin embargo, Guglielmelli et al. (2011) atribuyen al mayor contenido en TCT del heno en botón floral la menor DIVMO, mientras que la mayor DIVMO la encontraron al final de floración, que registraba el menor conte- 


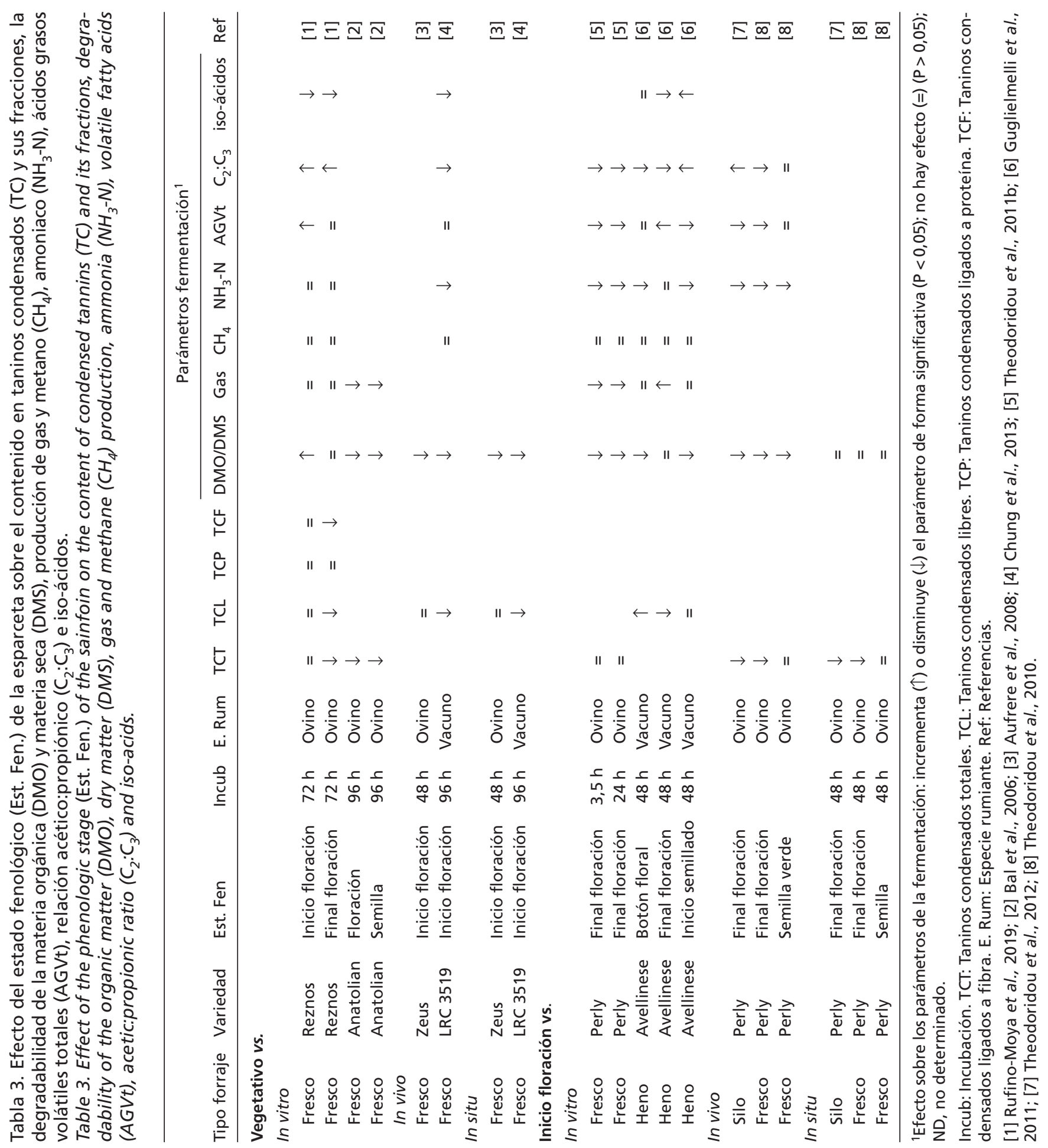


nido de TCT. Rufino-Moya et al. (2019b) observaron mayor DIVMO al inicio de floración que en el estado vegetativo o al final de la floración, lo cual no coincide ni con el contenido de TC ni de fibra, por lo que otros factores, como puede ser la estructura del TC, pueden influir en la fermentación. Theodoridou et al. (2011b) encontraron diferencias en DIVMO con similar contenido en fibras y en TCT, pero distinta actividad de los TC y grado de polimerización. La disparidad de resultados indica que otros factores diferentes al contenido en TC y en fibra, como pueden ser las características químicas de los TC o las condiciones en las que se realizan los ensayos de fermentación, afectan a la tasa de DIVMO.

El estado fenológico del forraje incubado puede afectar a la producción de gas aunque los resultados son contradictorios (Tabla 3 ). Bal et al. (2006) observaron una reducción de la producción de gas a medida que la esparceta maduraba, estando asociada a una menor DIVMO y fermentación en el rumen. En el mismo sentido, Theodoridou et al. (2011b) mostraron una reducción de la producción de gas al final de la floración respecto al inicio de floración de la esparceta, relacionado con más fibra, mayor grado de polimerización y menor actividad de los TC sin que existieran diferencias en el contenido en TC. Guglielmelli et al. (2011) no encontraron diferencias entre el estado inicio de floración y botón floral o inicio de formación de semilla, aunque si encontraron mayor producción de gas al final de la floración de la esparceta, la cual presentaba el menor contenido en TC respecto a los demás estados fenológicos. Contrariamente a los anteriores estudios, RufinoMoya et al. (2019b) no encontraron efecto del estado fenológico sobre la producción de gas al incubar esparceta fresca.

En general, los estudios muestran que el estado fenológico no afecta a la producción in vitro de $\mathrm{CH}_{4}$ independientemente del tiempo de incubación (Tabla 3), aunque los cambios en la porción fibrosa pueden provocar cambios en dicha producción (Beauchemin et al., 2008). No se ha visto efecto cuando la incubación in vitro se realizó sobre esparceta fresca durante $3,5 \mathrm{~h}$ y $24 \mathrm{~h}$ (Theodoridou et al., 2011b) ni en incubaciones más prolongadas (72 h) (Rufino-Moya et al., 2019b). Tampoco se observó dicho efecto cuando la incubación se realizó sobre heno de esparceta durante $48 \mathrm{~h}$ (Guglielmelli et al., 2011).

Varios estudios apuntan a diferencias en la producción de $\mathrm{NH}_{3}-\mathrm{N}$ entre los diferentes estados fenológicos debido a diferencias en el contenido en PB y/o a cambios en los TC. Durante el desarrollo de la planta se dan modificaciones del peso molecular de los TC, de la relación $\mathrm{PD}: \mathrm{PC}$, con un aumento de $\mathrm{PD}$, que favorece la interacción con las proteínas (Jonker y Yu, 2017) influyendo sobre su degradación ruminal y por tanto sobre el contenido en $\mathrm{NH}_{3}-\mathrm{N}$. Sin embargo, la mayoría de los estudios no determinan la estructura de los TC, por lo que es difícil saber la causa o causas de la variación del contenido en $\mathrm{NH}_{3}-$ $\mathrm{N}$. Theodoridou et al. (2011b) encontraron un menor contenido al final de la floración respecto al inicio de la floración cuando incubaron in vitro la esparceta fresca durante $3,5 \mathrm{~h}$ y $24 \mathrm{~h}$. En el mismo sentido, Guglielmelli et al. (2011) observaron un mayor contenido al inicio de floración respecto al final de floración en incubaciones in vitro de $48 \mathrm{~h}$ lo cual lo relacionaron con su mayor contenido en PB dado que el contenido en TC fue similar entre estados fenológicos. Dicha reducción de $\mathrm{NH}_{3}-\mathrm{N}$ a medida que avanza el desarrollo del cultivo se han observado en estudios in vivo realizados con esparceta en fresco (Chung et al., 2013), en heno (Theodoridou et al., 2010) y en silo (Theodoridou et al., 2012). Sin embargo, Rufino-Moya et al. (2019b) no encontraron ningún efecto del estado fenológico sobre el contenido de $\mathrm{NH}_{3}-\mathrm{N}$ cuando incubaron esparceta fresca en in vitro durante $72 \mathrm{~h}$, pudiendo estar asociado a las diferen- 
tes características de los TC, como se ha apuntado anteriormente, o al mayor tiempo de fermentación in vitro.

Los estudios del efecto del crecimiento del forraje sobre la producción total de AGV tampoco presentan unos resultados concluyentes (Tabla 3). Según Rufino-Moya et al. (2019b), la esparceta fresca al inicio de floración produce mayor cantidad de AGV que en estado vegetativo, mientras que no se registran diferencias cuando se estudia a final de floración. Otros estudios muestran que la producción de AGV es inferior a medida que avanza la madurez de la planta, tanto en estudios in vitro (Theodoridou et al., 2011b) como en estudios in vivo (Theodoridou et al., 2010 y 2012). Los resultados son contradictorios como demostraron Guglielmelli et al. (2011) en un mismo ensayo in vitro, donde observaron que la producción de AGV del heno de esparceta fue similar en las etapas de vegetativo y botón floral, mientras que al final de floración aumentaba y al inicio de formación de la semilla disminuía. Las discrepancias en el efecto del estado fenológico entre ensayos pueden ser debidos a la combinación de las diferencias en la composición química y en la estructura de los TC. En cuanto al efecto sobre la relación $C_{2}: C_{3}$, se ha observado su reducción con el desarrollo del cultivo, tanto en ensayos in vitro de incubación corta y media (hasta 48 h) (Guglielmelli et al., 2011; Theodoridou et al., 2011b) como en ensayos in vivo (Theodoridou et al., 2010; Chung et al., 2013). Sin embargo, en incubaciones más largas se encontró una mayor relación $\mathrm{C}_{2}: \mathrm{C}_{3}$ en la esparceta fresca en estado de inicio y final de floración que en el estado vegetativo Rufino-Moya et al. (2019b). Este incremento de la relación $C_{2}: C_{3}$ también se ha descrito en un ensayo in vivo con esparceta fresca al final de la floración respecto al inicio (Theodoridou et al., 2012). El estado fenológico también puede afectar a las proporciones de valérico e iso-ácidos debido a los cambios que se producen a medida que el forraje madura. Rufino-Moya et al. (2019b) observaron menor proporción de los iso-ácidos al inicio y al final de la floración y menor proporción del valérico al inicio de la floración respecto al estado vegetativo. Estos resultados van ligados tanto al contenido en PB de los forrajes como al contenido en TC. EI efecto reductor de los iso-ácidos a medida que madura la esparceta se ha descrito en un ensayo in vivo al comparar la esparceta al inicio con final de la floración (Chung et al., 2013). Sin embargo, en esparceta henificada, Guglielmelli et al. (2011) encontraron menores contenidos de iso-ácidos en la etapa de botón floral y final de floración y mayor contenido en inicio de formación de semilla respecto al inicio de floración.

\section{Efecto de la conservación de la esparceta sobre el contenido en taninos condensados y la fermentación ruminal}

Uno de los principales factores que también determinan el contenido de los TC y sus fracciones es la conservación del forraje (Tabla 4). La henificación del forraje puede dar lugar a la oxidación de los TC, pudiendo reducir su actividad biológica en función de la temperatura, la luz ultravioleta y el tiempo de secado a las que el forraje se expone (Wang et al., 2015). El efecto de la henificación varía en función de las condiciones del henificado. Cuando la henificación se realiza en campo se observa una reducción del $76 \%$ del contenido en TCT con respecto a la esparceta fresca (Aufrere et al., 2008). Cuando se realiza bajo condiciones experimentales controladas, únicamente se reducen un $28 \%$ los TCF (Rufino-Moya et al., 2019a) o se reducen las fracciones de TCP (56\%) y TCF (18\%) cuando la desecación se realiza con aire forzado a $30^{\circ} \mathrm{C}$ (Scharenberg et al., 2007a), pero no el contenido en TCT. El ensilado provoca 
Tabla 4. Contenido en taninos condensados totales (TCT), libres (TCL), ligados a la proteína (TCP) y ligados a fibra (TCF) de la esparceta según el método de conservación.

Table 4. Content of total condensed tannins (TCT), extractable (ECT), protein-bound (PBCT) and fiberbound (FBCT) of sainfoin according to the preservation method.

\begin{tabular}{|c|c|c|c|c|c|c|}
\hline Estado fenológico/variedad & Conservación & $\mathrm{TCT}$ & $\mathrm{TCL}$ & $\mathrm{TCP}$ & TCF & Ref \\
\hline \multicolumn{7}{|l|}{ Inicio floración } \\
\hline \multirow[t]{2}{*}{ LRC 3519} & Fresco & & 6,6 & & & [1] \\
\hline & Heno & & 5,5 & & & [1] \\
\hline \multirow[t]{3}{*}{ Visnovsky } & Fresco & 86,8 & 43,7 & 38 & 5,1 & [2] \\
\hline & Deshidratado & 66,5 & 46,2 & 17 & 3,7 & [2] \\
\hline & Silo & 78,9 & 28,8 & 46 & 4.2 & [2] \\
\hline \multirow[t]{2}{*}{ Zeus } & Fresco & & 25 & & & [3] \\
\hline & Heno & & 6 & & & [3] \\
\hline \multicolumn{7}{|l|}{ Floración } \\
\hline \multirow[t]{3}{*}{ Perly } & Fresco & 174,1 & 779 & 15 & 6 & [4] \\
\hline & Predesecado & 155,5 & 56 & 33 & 11 & [4] \\
\hline & Silo & 111,6 & 38 & 51 & 11 & [4] \\
\hline \multicolumn{7}{|l|}{ Final floración } \\
\hline \multirow[t]{3}{*}{ Cotswold Common } & Fresco & & 32,2 & 35 & & [5] \\
\hline & Predesecado & & 50,4 & 33 & & [5] \\
\hline & Silo & & 18 & 34 & & [5] \\
\hline \multirow[t]{3}{*}{ Perly } & Fresco & 207 & 79 & 15 & 6 & [4] \\
\hline & Pellets & 140,4 & 69 & 21 & 10 & [4] \\
\hline & Predesecado & 166,3 & 69 & 22 & 9 & [4] \\
\hline \multirow[t]{6}{*}{ Reznos } & Fresco & & 41,2 & 24 & & [5] \\
\hline & Predesecado & & 41,2 & 16 & & [5] \\
\hline & Silo & & 13,1 & 33 & & [5] \\
\hline & Fresco & 38,6 & 31,6 & 5,3 & 1,8 & [6] \\
\hline & Heno & 42,2 & 32,6 & 7,3 & 2,3 & [6] \\
\hline & Silo & 33,2 & 8,2 & 17 & 7,9 & [6] \\
\hline \multirow[t]{3}{*}{ Teruel } & Fresco & & 42,4 & 18 & & [5] \\
\hline & Predesecado & & 57,8 & 22 & & [5] \\
\hline & Silo & & 10,7 & 19 & & [5] \\
\hline \multicolumn{7}{|l|}{ No descrito } \\
\hline \multirow[t]{2}{*}{ Visnovsky } & Deshidratado & 77 & 50 & 21 & 6 & [7] \\
\hline & Silo & 71 & 5 & 47 & 19 & [7] \\
\hline
\end{tabular}

Ref: Referencias.

[1] Chung et al., 2014 [2] Scharenberg et al., 2007a ${ }^{1}$ [3] Aufrere et al., 2008 [4] Girard et al., 2018 [5] Lorenz et al., $2010^{2}$ [6] Rufino moya et al., 2019a² [7] Scharenberg et al., 2007b'.

${ }^{1} \mathrm{~g}$ eq TC Lotus pedunculatus/kg MS; ${ }^{2} \mathrm{~g}$ eq TC esparceta/kg MS. 
mayores cambios que la henificación, reduciendo el contenido de TCL y aumentando los de TCP y TCF por la ruptura parcial de las células de la planta que permite a los TCL interaccionar con otras moléculas presentes en el forraje (Wang et al., 2015; Mueller-Harvey et al., 2019). La interacción de los TC con las proteínas del forraje en el silo reduce la proteólisis que tiene lugar durante el proceso de ensilado, resultando una mejora de la calidad del silo (Theodoridou et al., 2012). Los efectos del ensilado sobre los TC y sus fracciones están determinados por la variedad del forraje (Lorenz et al. 2010), el estado fenológico (Girard et al., 2018) y/o el picado del forraje previo a su conservación (Scharenberg et al., 2007a; Girard et al., 2018). Generalmente, las fracciones de los TC varían más que los TCT, reduciéndose los TCL (45-77\%) en favor de los TCP y/o TCF (Scharenberg et al., 2007a; Lorenz et al., 2010; Girard et al., 2018; Rufino-Moya et al., 2019a). A pesar de que hay varios estudios que evalúan el efecto de la conservación sobre el contenido de TC y sus fracciones, únicamente hay dos estudios que comparen la fermentación de la esparceta según su conservación (Tabla 5).

El proceso de conservación de los forrajes influye en los parámetros de la fermentación a través de cambios en la cantidad de fibra y/o de cambios en las fracciones de los TC, que suelen ser más acusados en los ensilados. En este sentido, Rufino-Moya et al. (2019a) encontraron una reducción de la DIVMO con la henificación, pero no con el ensilado de esparceta con respecto al forraje fresco, presentando ambos forrajes conservados similar composición química, pero diferencias en las fracciones de TC. Las modificaciones que se registran durante el proceso del ensilado favorecen el ataque de los microorganismos ruminales (Arroquy et al., 2014) y por tanto no se registra una reducción de la DIVMO. Cuando se compara la esparceta deshidratada frente a la ensilada en un ensayo in vivo, la DMO fue inferior en la segunda (Scharenberg et al., 2007b), lo que pudo ser debido al proceso de ensilado per se y a la acción de los TC en el tracto digestivo.

Con respecto a la producción de gas y $\mathrm{CH}_{4}$, Rufino-Moya et al. (2019a) observaron un incremento de la producción de gas sin cambios en la de $\mathrm{CH}_{4}$ en la esparceta con la henificación, mientras que el ensilado redujo ambas producciones respecto a la esparceta fresca. Los autores relacionaron estos efectos con las fracciones de TCP y TCF, que protegen a la proteína y la fibra del ataque de los microorganismos con actividad proteolítica y fibrolítica (Bodas et al., 2012).

La conservación de la esparceta tiene escaso efecto sobre la producción de amoniaco y sobre la producción total de AGV (Scharenberg et al., 2007b; Rufino-Moya et al., 2019a) (Tabla 5). En cuanto a la relación $\mathrm{C}_{2}: \mathrm{C}_{3}$, RufinoMoya et al. (2019a) observaron que el ensilado de esparceta produjo una reducción de este parámetro mientras que la henificación no provocó diferencias respecto al forraje fresco. Tanto la henificación como el ensilado de la esparceta provocaron un incremento de los iso-ácidos respecto al forraje fresco independientemente de la reducción del contenido en la proteína (Rufino-Moya et al., 2019a), por lo que la causa de dicho incremento puede ser debido a modificaciones en las proporciones y estructura de los TC.

\section{Conclusiones}

El avance del estado fenológico y la conservación de la esparceta suelen reducir los contenidos de TC, dependiendo de las condiciones del cultivo, la variedad del forraje o del tipo de conservación. El ensilado genera cambios más importantes que el henificado, provocando una reducción de los TCL en favor de los TCP o TCF, reduciéndose en ocasiones los TCT. 


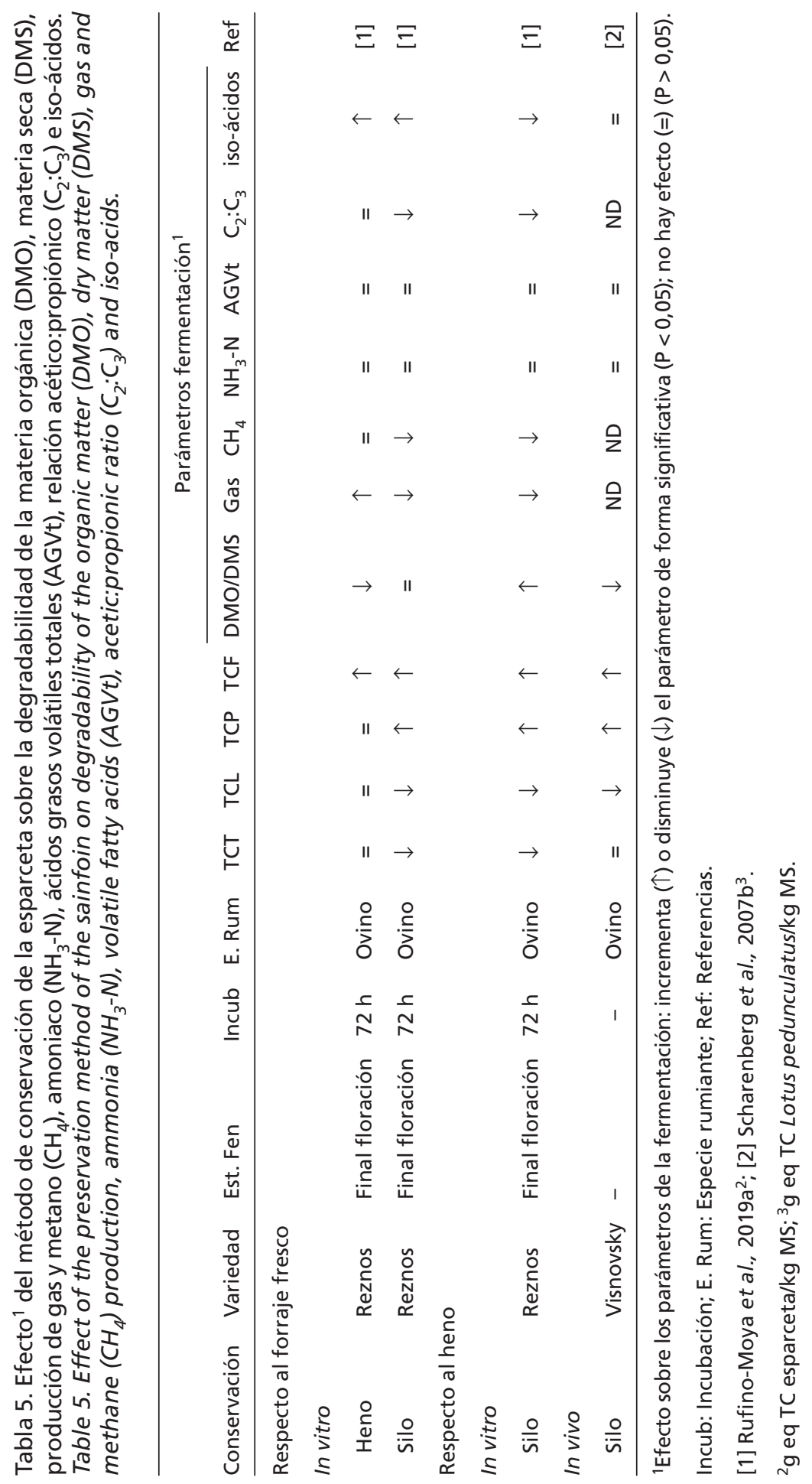


Los TC de la esparceta pueden provocar cambios beneficiosos en la fermentación, como la reducción de la producción de metano y de amoniaco. El avance del estado fenológico y de la conservación generan cambios en la composición química y en los taninos condensados que afectan a los parámetros de la fermentación ruminal. Con el avance de la madurez se reduce la degradabilidad de la MS o MO y el contenido en amoniaco, lo cual estaría más ligado al incremento de la fracción fibrosa y a la reducción de la proteína que al contenido en TC. Los efectos de la conservación dependen del método usado, tendiendo la henificación a reducir la degradabilidad más que el ensilado.

Es necesario que la comunidad científica acuerde unos criterios comunes en los ensayos de fermentación in vitro para que el forraje y sus TC sean los únicos factores que influyan en los resultados. Por otro lado, sería interesante realizar estudios más profundos de las características estructurales de los TC de la esparceta en sus diferentes variedades, estados fenológicos y métodos de conservación.

\section{Agradecimientos}

Este trabajo se ha realizado en el marco de los proyectos INIA-RTA2012-080-00 y RTA 2017-00008-C02-02, financiados por el MINECO y con el apoyo del Grupo de Investigación de Sistemas AgroGanaderos Alimentarios Sostenibles (SAGAS) del Gobierno de Aragón (A14-20R). P.J. Rufino-Moya tuvo un contrato FPI financiado por el INIA.

\section{Referencias bibliográficas}

Arroquy Jl, Cornacchione M, Colombatto D, Kunst Jr C (2014). Chemical composition and in vitro ruminal degradation of hay and silage from tropical grasses. Canadian Journal of Animal Science 94: 705-715. https://doi.org/10.4141/cjas-2014-014
Aufrère J, Dudilieu M, Poncet C (2008). In vivo and in situ measurements of the digestive characteristics of sainfoin in comparison with lucerne fed to sheep as fresh forages at two growth stages and as hay. Animal 2: 1331-1339. https://doi. org/10.1017/s1751731108002450

Bal MA, Ozturk D, Aydin R, Erol A, Ozkan CO, Ata M, Karakas E, Karabay P (2006). Nutritive value of sainfoin (Onobrychis viciaefolia) harvested at different maturity stages. Pakistan Journal of Biological Sciences 9: 205-209. https://doi.org/ 10.3923/pjbs.2006.205.209

Beauchemin KA, Kreuzer M, O'Mara F, McAllister TA (2008). Nutritional management for enteric methane abatement: A review. Australian Journal of Experimental Agriculture 48: 21-27. https://doi.org/10.1071/EA07199

Belenguer A, Hervás G, Toral PG, Fondevila M, Frutos $P$ (2011). Is polyethylene glycol innocuous to the rumen bacterial community? A preliminary in vitro study. Animal Production Science 51: 990-995. https://doi.org/10.1071/AN11041

Bodas R, Prieto N, García-González R, Andrés S, Giráldez FJ, López S (2012). Manipulation of rumen fermentation and methane production with plant secondary metabolites. Animal Feed Science and Technology 176: 78-93. https://doi. org/10.1016/j.anifeedsci.2012.07.010

Broadhurst RB, Jones WT (1978). Analysis of condensed tannins using acidified vanillin. Journal of the Science of Food and Agriculture 29: 788794. https://doi.org/10.1002/jsfa.2740290908

Brinkhaus AG, Wyss U, Arrigo Y, Girard M, Bee G, Zeitz JO, Kreuzer M, Dohme-Meier F (2017). In vitro ruminal fermentation characteristics and utilisable CP supply of sainfoin and birdsfoot trefoil silages and their mixtures with other legumes. Animal 11: 580-590. https://doi.org/10. 1017/S1751731116001816

Bueno ICS, Brandi RA, Franzolin R, Benetel G, Fagundes GM, Abdalla AL, Louvandini $\mathrm{H}$, Muir JP (2015). In vitro methane production and tolerance to condensed tannins in five ruminant species. Animal Feed Science and Technology 205: 1-9. https://doi.org/10.1016/j.anifeedsci. 2015.03.008 
Calabrò S, Guglielmelli A, lannaccone F, Danieli PP, Tudisco R, Ruggiero C, Piccolo G, Cutrignelli MI, Infascelli F (2012). Fermentation kinetics of sainfoin hay with and without PEG. Journal of Animal Physiology and Animal Nutrition 96: 842-849. https://doi.org/10.1111/j.1439-0396. 2011.01260.x

Chung YH, Mc Geough EJ, Acharya S, McAllister TA, McGinn SM, Harstad OM, Beauchemin KA (2013). Enteric methane emission, diet digestibility, and nitrogen excretion from beef heifers fed sainfoin or alfalfa. Journal of Animal Science 91: 48614874. https://doi.org/10.2527/jas.2013-6498

Delgado I, Andrés C, Muñoz F (2008). Effect of the environmental conditions on different morphological and agronomical characteristics of sainfoin. En: Sustainable Mediterranean grasslands and their multi-functions (Eds. Porqueddu C y Tavares de Sousa MM), Vol. 79, pp. 199-202. Zaragoza: CIHEAM / FAO / ENMP / SPPF.

Engstrom MT, Palijarvi M, Fryganas C, Grabber JH, Mueller-Harvey I, Salminen J-P (2014). Rapid qualitative and quantitative analyses of proanthocyanidin oligomers and polymers by UPLC-MS/MS. Journal of Agricultural and Food Chemistry 62: 3390-3399. https://doi.org/10. 1021/jf500745y

Frutos P, Hervás G, Ramos G, Giráldez F, Mantecón A (2002). Condensed tannin content of several shrub species from a mountain area in northern Spain, and its relationship to various indicators of nutritive value. Animal Feed Science and Technology 95: 215-226. https://doi.org/ 10.1016/S0377-8401(01)00323-6

Frutos P, Hervás G, Giráldez F, Mantecón A (2004a). Review. Tannins and ruminant nutrition. Spanish Journal of Agricultural Research 2: 191202. http://dx.doi.org/10.5424/sjar/2004022-73

Frutos P, Hervás G, Giráldez FJ, Mantecón AR (2004b). An in vitro study on the ability of polyethylene glycol to inhibit the effect of quebracho tannins and tannic acid on rumen fermentation in sheep, goats, cows, and deer. Australian Journal of Agricultural Research 55: 1125-1132. https://doi.org/10.1071/ar04058

Gea A, Stringano E, Brown RH, Mueller-Harvey I (2011). In situ analysis and structural elucidation of sainfoin (Onobrychis viciifolia) tannins for high-throughput germplasm screening. Journal of Agricultural and Food Chemistry 59: 495503. https://doi.org/10.1021/jf103609p

Getachew G, Blümmel M, Makkar HPS, Becker K (1998). In vitro gas measuring techniques for assessment of nutritional quality of feeds: A review. Animal Feed Science and Technology 72: 261-281. https://doi.org/10.1016/S0377-8401 (97)00189-2

Getachew G, Makkar HPS, Becker K (2000). Effect of polyethylene glycol on in vitro degradability of nitrogen and microbial protein synthesis from tannin-rich browse and herbaceous legumes. British Journal of Nutrition 84: 73-83. https://doi.org/10.1017/S0007114500001252

Girard M, Dohme-Meier F, Ampuero-Kragten S, Grosse-Brinkhaus A, Arrigo Y, Wyss U, Bee G (2018). Modification of the proportion of extractable and bound condensed tannins in birdsfoot trefoil (Lotus corniculatus) and sainfoin (Onobrychis viicifolia) during wilting, ensiling and pelleting processes. Biotechnology in Animal Husbandry 34: 1-19. https://doi.org/10. 2298/BAH1801001G

Guglielmelli A, Calabrò S, Primi R, Carone F, Cutrignelli MI, Tudisco R, Piccolo G, Ronchi B, Danieli PP (2011). In vitro fermentation patterns and methane production of sainfoin (Onobrychis viciifolia scop.) hay with different condensed tannin contents. Grass and Forage Science 66: 488-500. https://doi.org/10.1111/j.1365-24 94.2011.00805.x

Hatew B, Hayot Carbonero C, Stringano E, Sales LF, Smith LMJ, Mueller-Harvey I, Hendriks WH, PeIlikaan WF (2015). Diversity of condensed tannin structures affects rumen in vitro methane production in sainfoin (Onobrychis viciifolia) accessions. Grass and Forage Science 70: 474490. https://doi.org/10.1111/gfs. 12125

Hatew B, Stringano E, Mueller-Harvey I, Hendriks WH, Carbonero CH, Smith LMJ, Pellikaan WF (2016). Impact of variation in structure of condensed tannins from sainfoin (Onobrychis viciifolia) on in vitro ruminal methane production and fermentation characteristics. Journal of Animal Physiology and Animal Nutrition 100: 348-360. https://doi.org/10.1111/jpn.12336 
Heinritz SN, Martens SD, Avila P, Hoedtke S (2012). The effect of inoculant and sucrose addition on the silage quality of tropical forage legumes with varying ensilability. Animal Feed Science and Technology 174: 201-210. https://doi.org/ 10.1016/j.anifeedsci.2012.03.017

Hervás G, Frutos P, Giráldez FJ, Mantecón ÁR, Álvarez Del Pino MC (2003). Effect of different doses of quebracho tannins extract on rumen fermentation in ewes. Animal Feed Science and Technology 109: 65-78. https://doi.org/10.1016/ S0377-8401(03)00208-6

Huyen N, Fryganas C, Uittenbogaard G, MuellerHarvey I, Verstegen M, Hendriks W, Pellikaan W (2016). Structural features of condensed tannins affect in vitro ruminal methane production and fermentation characteristics. The Journal of Agricultural Science 154: 1474-1487. https:// doi.org/10.1017/S0021859616000393

Jayanegara A, Goel G, Makkar HPS, Becker K (2015). Divergence between purified hydrolysable and condensed tannin effects on methane emission, rumen fermentation and microbial population in vitro. Animal Feed Science and Technology 209: 60-68. https://doi.org/ 10.1016/j.anifeedsci.2015.08.002

Jonker A, Yu P (2017). The occurrence, biosynthesis, and molecular structure of proanthocyanidins and their effects on legume forage protein precipitation, digestion and absorption in the ruminant digestive tract. International Journal of Molecular Sciences 18: 1105. https://doi.org/ 10.3390/ijms18051105

Kelman W, Tanner G (1990). Foliar condensed tannin levels in lotus species growing on limed and unlimed soils in south-eastern Australia. Proceedings of the New Zealand Grasslands Association 52: 51-54.

Kumar R, Singh M (1984). Tannins: Their adverse role in ruminant nutrition. Journal of Agricultural and Food Chemistry 32: 447-453. https:// doi.org/10.1021/jf00123a006

Li Y, Iwaasa A, Wang Y, Jin L, Han G, Zhao M (2014). Condensed tannins concentration of selected prairie legume forages as affected by phenological stages during two consecutive growth seasons in western canada. Canadian Journal of Plant Science 94: 817-826. https://doi. org/10.4141/cjps2013-234

Lorenz MM, Eriksson T, Udén P (2010). Effect of wilting, silage additive, PEG treatment and tannin content on the distribution of $\mathrm{N}$ between different fractions after ensiling of three different sainfoin (Onobrychis viciifolia) varieties. Grass and Forage Science 65: 175-184. https:// doi.org/10.1111/j.1365-2494.2010.00736.x

Makkar H (2003). Effects and fate of tannins in ruminant animals, adaptation to tannins, and strategies to overcome detrimental effects of feeding tannin-rich feeds. Small Ruminant Research 49: 241-256. https://doi.org/10.1016/S0921-4488 (03)00142-1

Martínez TF, McAllister TA, Wang Y, Reuter T (2006). Effects of tannic acid and quebracho tannins on in vitro ruminal fermentation of wheat and corn grain. Journal of the Science of Food and Agriculture 86: 1244-1256. https://doi. org/10.1002/jsfa.2485

McMahon L, McAllister T, Berg B, Majak W, Acharya S, Popp J, Coulman B, Wang Y, Cheng K-J (2000). $A$ review of the effects of forage condensed tannins on ruminal fermentation and bloat in grazing cattle. Canadian Journal of Plant Science 80: 469-485. https://doi.org/10.4141/P99-050

Ministerio de Agricultura, Pesca y Alimentacion (MAPA) (2019). Superficies y producciones de cultivos. 7.5.11.3. Cultivos forrajeros-leguminosas forrajeras-esparceta: Análisis provincial de superficie, rendimiento y producción en verde, 2018. Disponible en https://www.mapa. gob.es/estadistica/pags/anuario/2019-Avance/ CAPITULOSPDF/CAPITULO07/pdfc07_5.11.3.pdf (consultado: 2 agosto 2020.

Mole S, Ross JA, Waterman PG (1988). Light-induced variation in phenolic levels in foliage of rain-forest plants. Journal of Chemical Ecology 14: 23-34. https://doi.org/10.1007/BF01022528

Mueller-Harvey I (2006). Unravelling the conundrum of tannins in animal nutrition and health. Journal of the Science of Food and Agriculture 86: 2010-2037. https://doi.org/10.1002/jsfa.2577

Mueller-Harvey I, Bee G, Dohme-Meier F, Hoste H, Karonen M, Kölliker R, Lüscher A, Niderkorn V, 
Pellikaan WF, Salminen J-P, Skøt L, Smith LMJ, Thamsborg SM, Totterdell P, Wilkinson I, WiIliams AR, Azuhnwi BN, Baert N, Brinkhaus AG, Copani G, Desrues O, Drake C, Engström M, Fryganas C, Girard M, Huyen NT, Kempf K, Malisch C, Mora-Ortiz M, Quijada J, Ramsay A, Ropiak HM, Waghorn GC (2019). Benefits of condensed tannins in forage legumes fed to ruminants: Importance of structure, concentration, and diet composition. Crop Science 59: 125. https://doi.org/10.2135/cropsci2017.06.0369

Niderkorn V, Mueller-Harvey I, Le Morvan A, Aufrère J (2012). Synergistic effects of mixing cocksfoot and sainfoin on in vitro rumen fermentation. Role of condensed tannins. Animal Feed Science and Technology 178: 48-56. https:// doi.org/10.1016/j.anifeedsci.2012.09.014

O'Donovan L, Brooker JD (2001). Effect of hydrolysable and condensed tannins on growth, morphology and metabolism of Streptococcus gaIlolyticus (S. caprinus) and Streptococcus bovis. Microbiology 147: 1025-1033. https://doi.org/ 10.1099/00221287-147-4-1025

Patra AK, Saxena J (2011). Exploitation of dietary tannins to improve rumen metabolism and ruminant nutrition. Journal of the Science of Food and Agriculture 91: 24-37. https://doi.org/ 10.1002/jsfa.4152

Piluzza G, Bullitta S (2010). The dynamics of phenolic concentration in some pasture species and implications for animal husbandry. Journal of the Science of Food and Agriculture 90: 1452-1459. https://doi.org/10.1002/jsfa.3963

Piluzza G, Sulas L, Bullitta S (2014). Tannins in forage plants and their role in animal husbandry and environmental sustainability: A review. Grass and Forage Science 69: 32-48. https://doi. org/10.1111/gfs. 12053

Porter LJ, Hrstich LN, Chan BG (1985). The conversion of procyanidins and prodelphinidins to cyanidin and delphinidin. Phytochemistry 25: 223-230. https://doi.org/10.1016/S0031-9422(00) 94533-3

Rufino-Moya P, Blanco M, Bertolín J, Joy M (2019a). Effect of the method of preservation on the chemical composition and in vitro fermentation characteristics in two legumes rich in con- densed tannins. Animal Feed Science and Technology 251: 12-20. https://doi.org/10.1016/j.ani feedsci.2019.02.005

Rufino-Moya PJ, Blanco M, Bertolín JR, Joy M (2019b). Methane production of fresh sainfoin, with or without PEG, and fresh alfalfa at different stages of maturity is similar but the fermentation end products vary. Animals 9: 197. https://doi.org/10.3390/ani9050197

Scharenberg A, Arrigo Y, Gutzwiller A, Soliva CR, Wyss U, Kreuzer M, Dohme F (2007a). Palatability in sheep and in vitro nutritional value of dried and ensiled sainfoin (Onobrychis viciifolia) birdsfoot trefoil (Lotus corniculatus), and chicory (Cichorium intybus). Archives of Animal Nutrition 61: 481-496. https://doi.org/10. 1080/17450390701664355

Scharenberg A, Arrigo Y, Gutzwiller A, Wyss U, Hess HD, Kreuzer M, Dohme F (2007b). Effect of feeding dehydrated and ensiled tanniferous sainfoin (Onobrychis viciifolia) on nitrogen and mineral digestion and metabolism of lambs. Archives of Animal Nutrition 61: 390-405. https://doi.org/10.1080/17450390701565081

Stringano E, Cramer R, Hayes W, Smith C, Gibson T, Mueller-Harvey I (2011). Deciphering the complexity of sainfoin (Onobrychis viciifolia) proanthocyanidins by MALDI-TOF mass spectrometry with a judicious choice of isotope patterns and matrixes. Analytical chemistry 83: 4147-4153. https://doi.org/10.1021/ac2003856

Terrill T, Rowan A, Douglas G, Barry T (1992). Determination of extractable and bound condensed tannin concentrations in forage plants, protein concentrate meals and cereal grains. Journal of the Science of Food and Agriculture 58: 321329. https://doi.org/10.1002/jsfa.2740580306

Theodoridou K, Aufrère J, Andueza D, Pourrat J, Le Morvan A, Stringano E, Mueller-Harvey I, Baumont R (2010). Effects of condensed tannins in fresh sainfoin (Onobrychis viciifolia) on in vivo and in situ digestion in sheep. Animal Feed Science and Technology 160: 23-38. https://doi. org/10.1016/j.anifeedsci.2010.06.007

Theodoridou K, Aufrere J, Andueza D, Le Morvan A, Picard F, Stringano E, Pourrat J, Mueller-Harvey I, Baumont R (2011a). Effect of plant deve- 
lopment during first and second growth cycle on chemical composition, condensed tannins and nutritive value of three sainfoin (Onobrychis viciifolia) varieties and lucerne. Grass and Forage Science 66: 402-414. https://doi.org/ 10.1111/j.1365-2494.2011.00798.x

Theodoridou K, Aufrère J, Niderkorn V, Andueza D, Le Morvan A, Picard F, Baumont R (2011b). In vitro study of the effects of condensed tannins in sainfoin on the digestive process in the rumen at two vegetation cycles. Animal Feed Science and Technology 170: 147-159. https:// doi.org/10.1016/j.anifeedsci.2011.09.003

Theodoridou K, Aufrére J, Andueza D, Le Morvan A, Picard F, Pourrat J, Baumont R (2012). Effects of condensed tannins in wrapped silage bales of sainfoin (Onobrychis viciifolia) on in vivo and in situ digestion in sheep. Animal 6: 245-253. https://doi.org/10.1017/S1751731111001510

Tiemann TT, Avila P, Ramírez G, Lascano C, Kreuzer $\mathrm{M}$, Hess $\mathrm{H}$ (2008). In vitro ruminal fermentation of tanniniferous tropical plants: Plant-specific tannin effects and counteracting efficiency of
PEG. Animal Feed Science and Technology 146: 222-241. https://doi.org/10.1016/j.anifeedsci. 2007.12.009

Toral PG, Hervás G, Missaoui H, Andrés S, Giráldez FJ, Jellali S, Frutos P (2016). Effects of a tanninrich legume (Onobrychis viciifolia) on in vitro ruminal biohydrogenation and fermentation. Spanish Journal of Agricultural Research 14: 19. https://doi.org/10.5424/sjar/2016141-8989

Waghorn G (2008). Beneficial and detrimental effects of dietary condensed tannins for sustainable sheep and goat production-progress and challenges. Animal Feed Science and Technology 147: 116-139. https://doi.org/10.1016/j.anifeedsci.2007.09.013

Wang Y, McAllister TA, Acharya S (2015). Condensed tannins in sainfoin: Composition, concentration, and effects on nutritive and feeding value of sainfoin forage. Crop Science 55: 13-22. https://doi.org/10.2135/cropsci2014.07.0489

(Aceptado para publicación el 3 de agosto de 2020) 